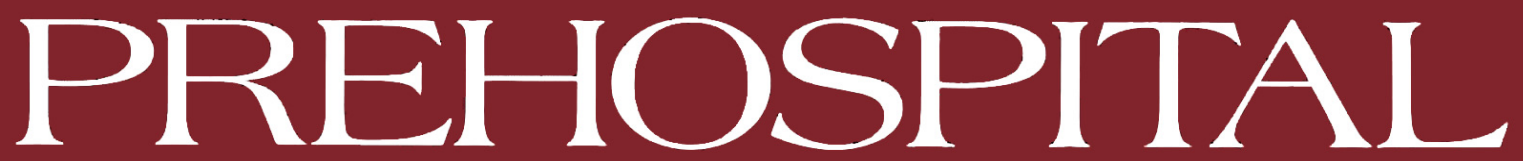

\title{
and
}
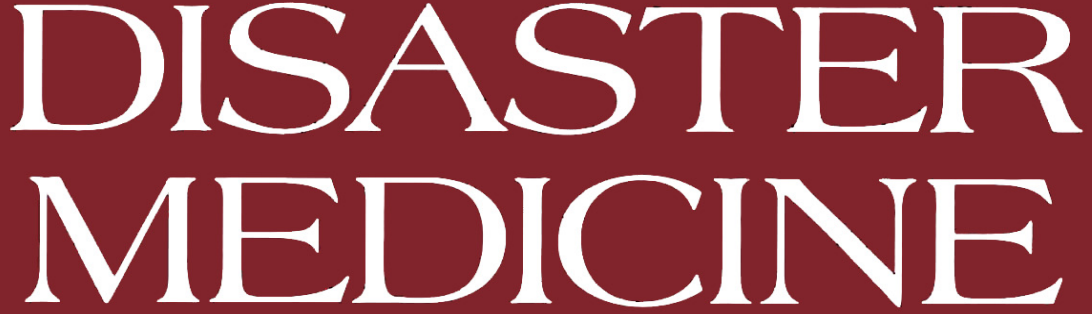

Médecine Pré-Hospitalière et Médecine de Catastrophe

Medicina Prehospitalária y de Catástrofes

病院にかかる前の処置と

桨害运㾦

Volume 14, Number 4

October-December 1999

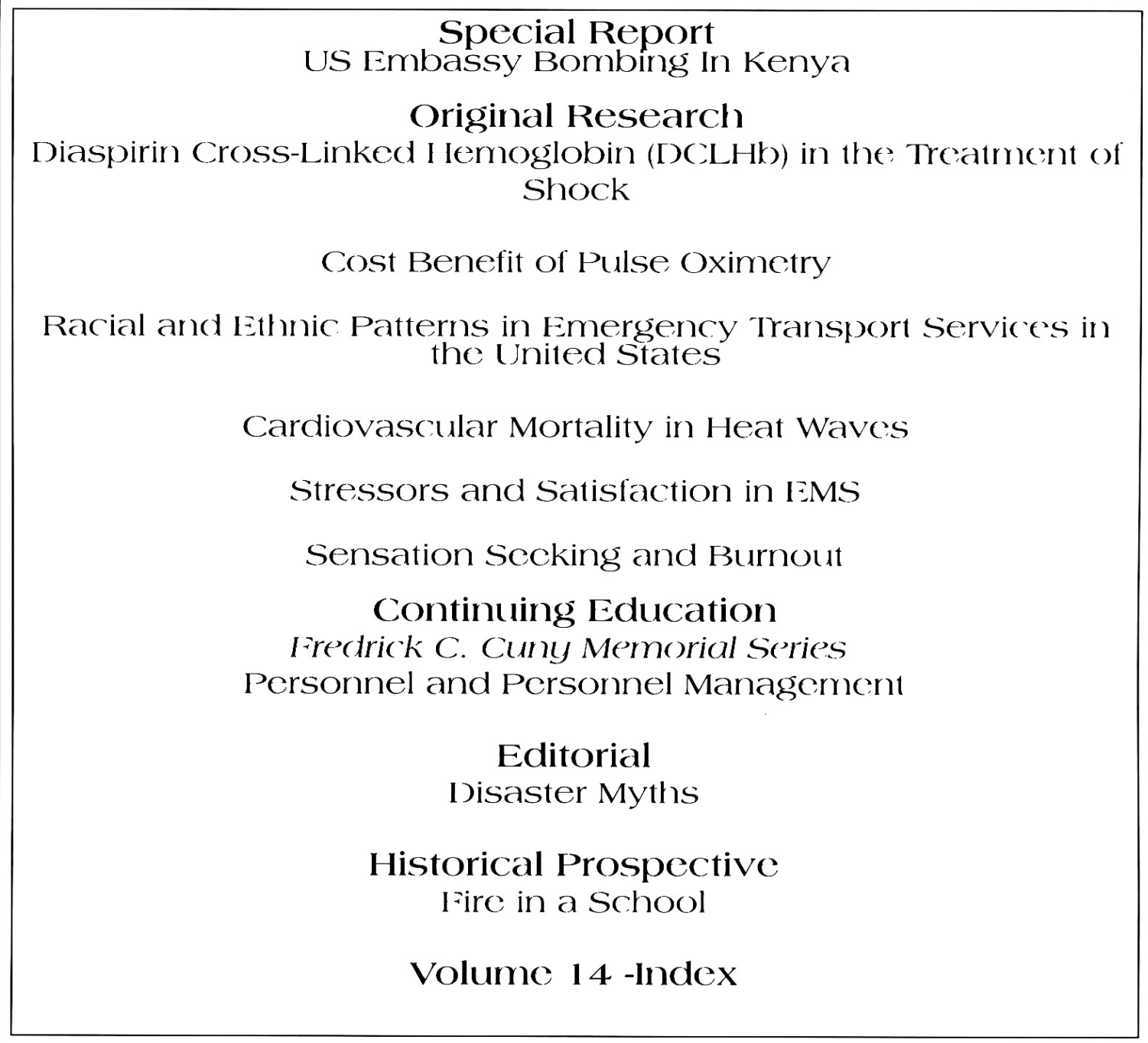

The Official Journal of the

World Association for Disaster and Emergency Medicine

and the

Nordic Seriety of Disaster Medicine 


\section{To the Rescue}
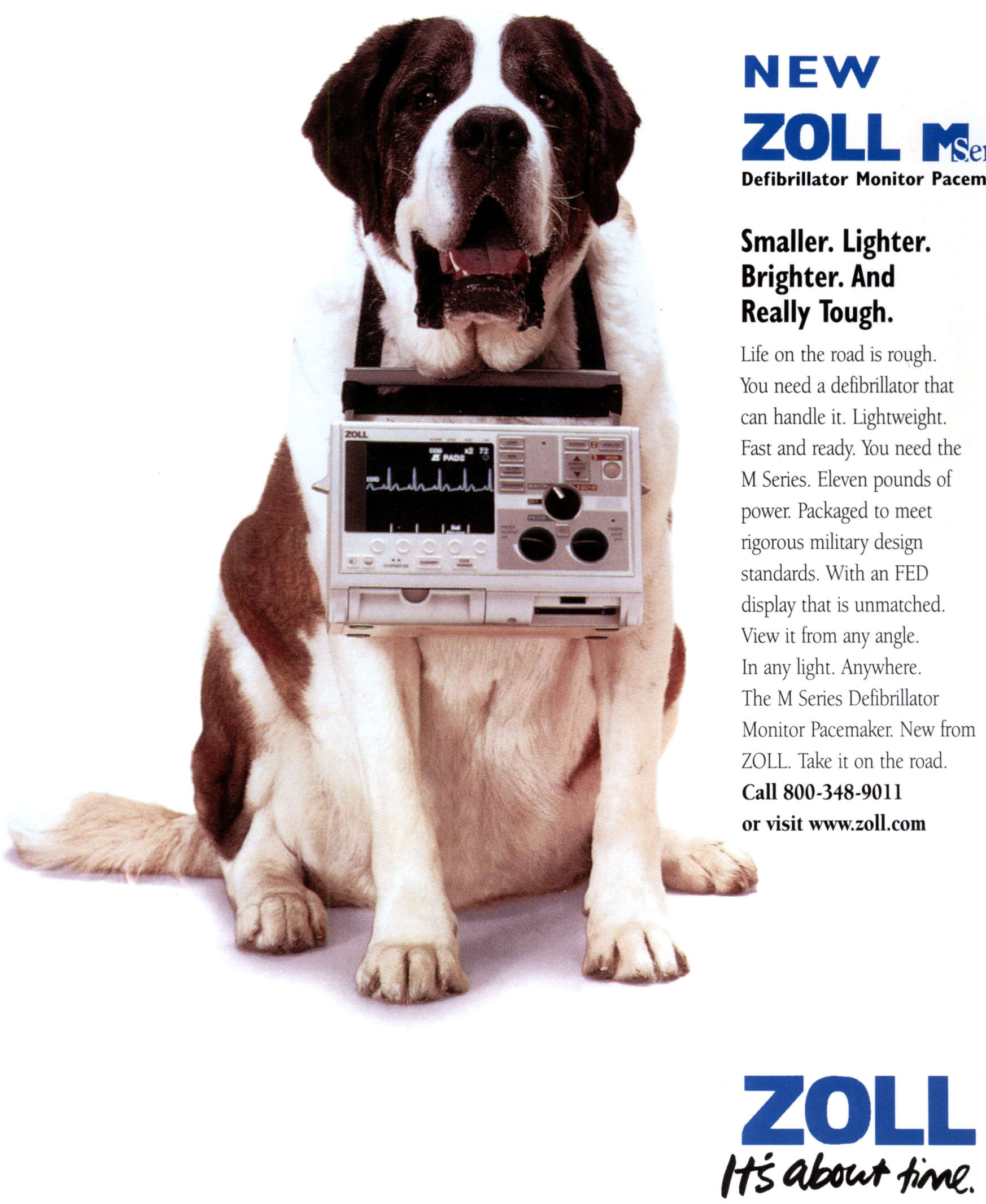


\section{Prehospital and Disaster Medicine Official Journal of the World Association for Disaster and Emergency Medicine and the Nordic society of Disaster Medicine}

Each Issue Brings:

- Original research by international authors in the sciences of emergency, disaster and humanitarian medicine and management

- Summaries and critiques of published research

- Analysis of emergency and disaster medicine services

- Abstracts from important meetings and congresses

- Continuing education

- Free access to Internet version

\section{I'11}

YES!

\section{Individual}

Institutional
Subscribe!

1 year (4 Issues)

$\$ 55$

$\$ 78$
Save

$15 \%$ !

2 years ( 8 Issues) 3 years (12 Issues)

$\$ 94$

$\$ 132$
Save

$25 \%$ !

$\$ 124$

$\$ 175$

$\square$ Check here if entitled to the $25 \%$ discount as member of affiliated organizations

Organization

$\square$ Payment Enclosed $\quad \square$ Bill Me $\square$ Visa $\square$ MasterCard

Card\# Exp. Date

Signature

Name

Title

Address

City

State___ Z Z Z _ _

Zip

Outside U.S. add $\$ 16$ per subscription per year.

\section{Get Your Subscription to Prehospital and Disaster Medicine FREE!}

\section{Here's how.}

Join the World Association for Disaster and Emergency Medicine for only $\$ 120$ per year and receive:

- Subscription to Prehospital and Disaster Medicine

- Preferred rates for registration at the World and Regional Congresses

- Semi-annual newsletter

- Membership directory

- Special Affiliated Society Membership advantages $\square$ Yes, I'll Join! ๑ Please Send More Information

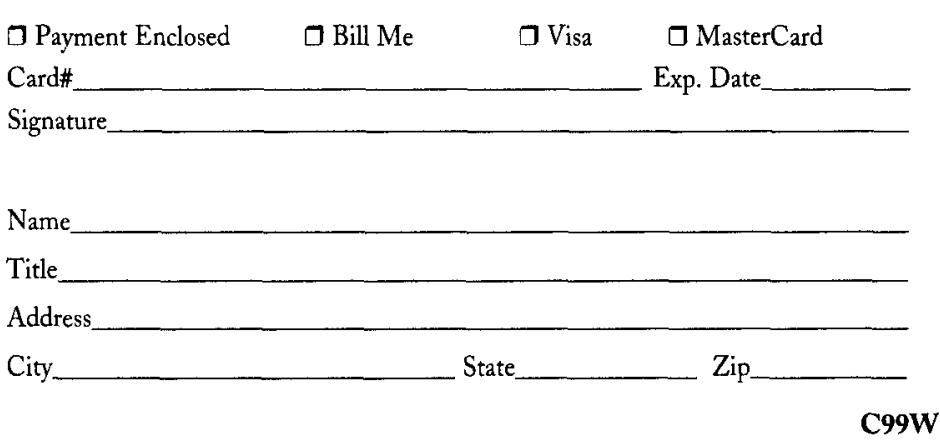




\section{AIR MAIL PAR AVION}

IBRS/CCRI No. 1780
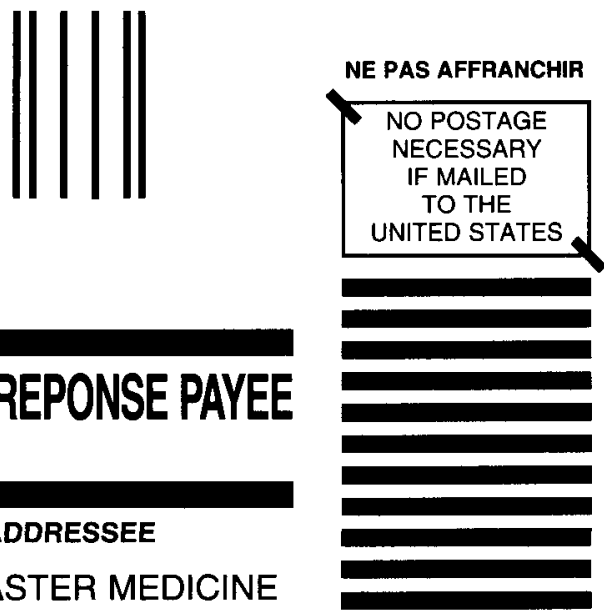

E5/615 CLINICAL SCIENCE CENTER

600 HIGHLAND AVENUE

MADISON WI 53791-9744

UNITED STATES OF AMERICA

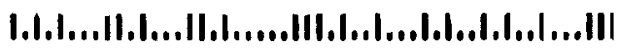

\section{AIR MAIL \\ PAR AVION}

IBRS/CCRI No. 1780
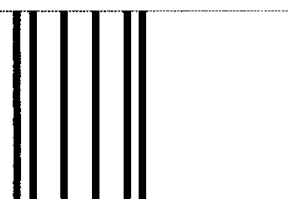

INTERNATIONAL BUSINESS REPLY MALLREPONSE PAYEE

POSTAGE WILL BE PAID BY ADDRESSEE

WADEM

E5/615 CLINICAL SCIENCE CENTER

600 HIGHLAND AVENUE

MADISON WI 53791-9744

UNITED STATES OF AMERICA

$$
\text { I.I,I...II.I...II,I.....III.I..I...I.I.,I,I.,I...III }
$$

NE PAS AFFRANCHIR

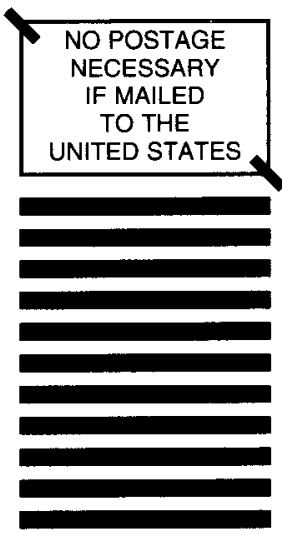




\section{Table of Contents}

Editor's Corner

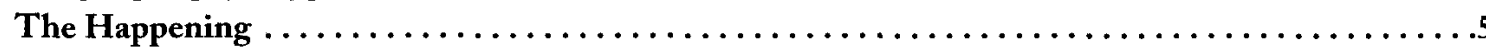

Marvin L. Birnbaum, $M D, P h D$

\section{Editorial}

Stop Propagating Disaster Myths $\ldots \ldots \ldots \ldots \ldots \ldots \ldots \ldots \ldots \ldots \ldots \ldots \ldots \ldots \ldots$

Dr. Claude de Ville de Goyet

\section{Special Reports}

The International Search and Rescue Response to the US Embassy Bombing in Kenya:

The Medical Team Experience $\ldots \ldots \ldots \ldots \ldots \ldots \ldots \ldots \ldots \ldots$
Anthony G. Macintyre $M D$; Scott Weir, $M D ;$ Joseph $A$. Barbera, $M D$

Original Research

Cardiovascular Mortality - the Hidden Peril of Heat Waves ....................

Sherrilyn H. Wainwright, DVM, MPH; Sharunda D. Buchanan, MS, PhD; M. Mainzer, DVM, MS;

R. Gibson Parrish, MD; Thomas H. Sinks, PhD

Racial and Ethnic Patterns in the Utilization of Prehospital Emergency Transport Services

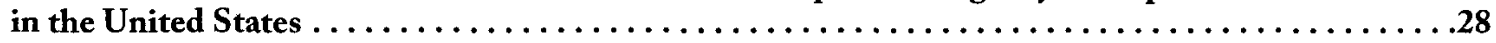
Charles E. McConnel, $P h D$; Rosemary W. Wilson, $P h D$

Job Stressors and Job Satisfaction in a Major Metropolitan Public EMS Service $\ldots \ldots \ldots \ldots . . .32$ J. Shelby Bowron, MD, MPH, FACEP; Knox H. Todd, MD, MPH

Sensation Seeking as It Relates to Burnout Among Emergency Medical Personnel: A Texas Study .36 Chwee Lye Chng, PhD; Starr Eaddy, MS, EMT

The Cost-Benefit of Pulse-Oximeter Use in the Prehospital Environment $\ldots \ldots \ldots \ldots \ldots \ldots 41$ Andrew J. Macnab, MD, FRCPC; Lark Susak, RN, BScN; Faith A. Gagnon, HBSc; Janet Alred; Charles Sun, MD, FRCPC

A Safety Assessment of Diaspirin Cross-Linked Hemoglobin (DCLHb) in the Treatment of Hemorrhagic, Hypovolemic Shock .............................. Robert J. Przybelski; Elaine K. Daily; Jean Micheels; Edward Sloan; Pierre Mols; Luc Corne; Max D. Koenigsberg; William H. Bickell; Dan R. Thompson; Jay D. Harviel; Stephen M. Cohn

Historical Perspective

Collinwood: An Anatomy of a Disaster ...

Paul Rega 


\section{Continuing Education}

Fredrick C. Cuny Memorial Series

Principles of Disaster Management

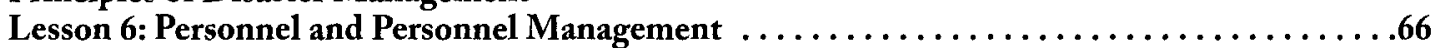

Fred C. Cuny

\section{Index}

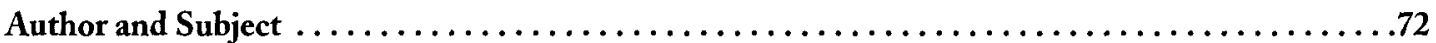

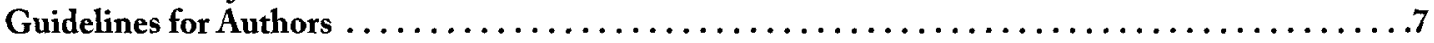

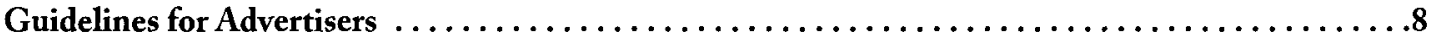

\section{Editorial Office}

University of Wisconsin-Madison USA

Marvin L. Birnbaum, MD, PhD, Editor-in-Chief

Associate Editor

Mary Elizabeth Braun

Production Editor

Donna Schaan

Web Production Editor

Megan Phillips

University of Wisconsin School of Jounalism

Student Interns

Krista de Bellis

Anne Marie Caremona

\section{Advertising}

Mary Duss

Subscriptions

Mary Elizabeth Braun

Editorial Office

University of Wisconsin-Madison

\section{Publisher}

World Association for Disaster and

Emergency Medicine

Madison, Wisconsin USA
Prehospital and Disaster Medicine (ISSN 1049-023X) is published quarterly in the months of January, April, July, and October by the World Association for Disaster and Emergency Medicine. Prebospital and Disaster Medicine incorporates the Journal of the World Association for Emergency and Disaster Medicine and the Joumal of Prehospital Medicine.

Editorial Information: Submit manuscripts and editorial inquiries to: Marvin L. Birnbaum, $\mathrm{PhD}, \mathrm{MD}$, Editor, Prehospital and Disaster Medicine, Clinical Science Center, $600 \mathrm{~N}$. Highland (E5/615), Madison, WI 53792, USA; Telephone/Facsimile (01) (608) 263-9641; E-mail: mlb@medicine.wisc.edu

Subscription Information: One year (four issues)-Institutions: (U.S.) \$78; Individuals: $\$ 55$; Canadian and international subscribers must add $\$ 13$ per year for postage. Claims of non-receipt or damaged issues must be filed within three months of cover date. POSTMASTER: Send address changes to Prehospital and Disaster Medicine, Clinical Science Center, 600 N. Highland (E5/615), Madison, WI 53792 USA.

Advertising Information: Mary Duss; Prehospital and Disaster Medicine, Clinical Science Center, $600 \mathrm{~N}$. Highland (E5/615), Madison, WI 53792 USA

Abstracts: Comprehensively abstracted by the National Library of Medicine (Medline), Cumulative Index to Nursing and Allied Health (CINAHL) and Health Star Cumulative Index. The database is available online via BRS, DataStar, and DIALOG, and on CD-ROM through CD Plus, Compact Cambridge and Silver Platter. Abstracts and Search capability available on the Internet at: http://pdm.medicine.wisc.edu.

Copyright $(\mathcal{O} 1999$ by the World Association for Disaster and Emergency Medicine.

Periodicals postage paid at Madison, WI and at additional mailing offices

Bulk Rate U.S.
Fostage PAID
Ephrata, PA
Perrit No. 17708




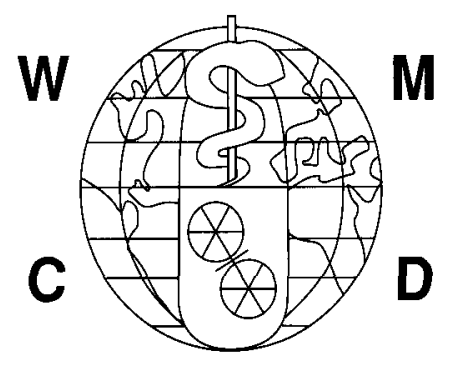

E

$12^{\text {th }}$ World Congress

on

Disaster and Emergency Medicine

WADEM

World Association for Disaster and Emergency Medicine

\section{May 9 - 12, 2001 \\ LYON, FRANCE}

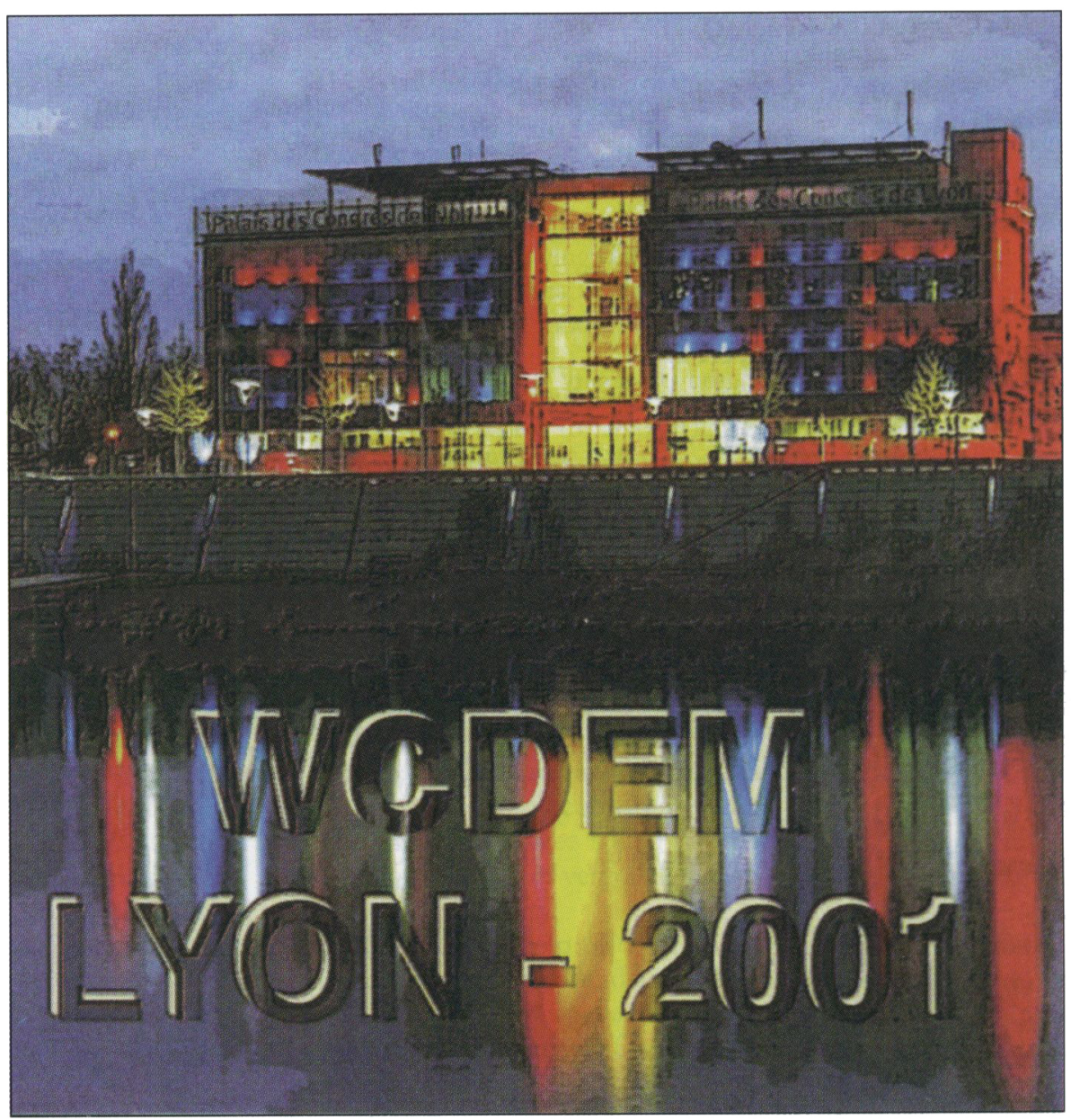


EDITOR-IN-CHIEF

Marvin L. Birnbaum, MD, PhD

University of Wisconsin-Madison

Madison, Wisconsin USA

\section{ASSOCIATE EDITORS}

Wolfgang Dick, Dr Med

Johannes Gutenberg Universität

Mainz, Germany

Kimball Maull, MD

Carraway Injury control Institute

Birningham, Alabama USA

Ernesto A. Pretto, MD

University of Pittsburgh

Pittsburgh, Pennsylvania USA

Steven Rottman, MD

University of CaliforniaLos Angeles

Los Angeles, California USA

\section{SECTION EDITORS}

Terrorism

Michael Moles, MD

Prince Philip Hospital

Hong Kong University

Hong Kong

\section{PAST EDITORS}

R Adams Cowley, MD

Deceased

(JWAEDM 1986-1987)

\section{Peter Safar, MD}

Safar Center

University of Pittsburgh

Pittsburgh, Pennsylvania USA

(JWAEDM 1981-1985)

Michael Gunderson, REMTP

Institute for Prehospital

Medicine

Tempe, Arizona USA

\section{CONSULTING EDITORS}

S. William A. Gunn, MD

European Centre for Disaster Medicine

Geneva, Switzerland

Peter Safar, MD

Safar Center

University of Pittsburgh

Pittsburgh, Pennsylvania USA

Martin Silverstein, MD

Uniformed Services University of the Health Sciences

Bethesda, Maryland USA

\section{Ronald Stewart, MD}

Victoria Hospital

Halifax, Nova Scotia Canada

\section{EDITORIAL BOARD}

Jakov Adler, MD

Jerusalem, Israel

\section{Ahmed Ammar, MD \\ King Fahd University Hospital \\ Al-Rhobar, Saudi Arabia}

V. Anantharaman, MD

Singapore General Hospital

Singapore

Paul B. Anderson

Lincoln Medical Education Foundation

Rural EMS Institute

Lincoln, Nebraska USA

Bishara Atiyeh, MD

American University

Beirut, Lebanon

Jack Ayres, JD, REMT-P

University of Texas Health Science Center

Dallas, Texas USA

Jan Babik, MD

Kosice Burn Center

Kosice-Saca, Slovakia

Peter Baskett, MD

Frenchay Hospital

Bristol, UK

Richard A. Bissell, PhD

University of MarylandBaltimore Campus

Baltimore, Maryland USA

Fredrick M. Burkle, MD, MPH

John A. Burns School of Medicine and School of Public Health

University of Hawaii

Honolulu, Hawaii USA

Jeff Clawson, MD

Medical Priority Consultants,

Salt Lake City, Utah USA

Richard O. Cummins, MD

University of Washington

Seattle, Washington USA

Craig DeAtley, PA-C

George Washington University Medical Center

Washington, D.C. USA

Claude de Ville de Goyet, MD

Pan-American Health Organization, World Health Organization

James DuCharme, MD

Royal Victoria Hospital

Montréal, Quèbec Canada

Judith Fisher, MD

British Association for Immediate Care

London, UK

Malcolm Fisher, MD

Royal North Shore Hospital

Sydney, Australia

Alain Flaujat, MD

Editor, Amelisap

Olonzao, France

Erik S. Gaull

TriData Corporation

Arlington, Virginia USA

Sergey Goncharov, MD

All-Russian Disaster Medicine Center

Moscow, Russia
Michael Gunderson, REMT-P James O. Page, JD

Institute for Prehospital Medicine

Tempe, Arizona USA

Borje Hallen, MD

Karolinska Sjukhuset

Stockholm, Sweden

Connie A. Jastremski, RN, MS

Syracuse, New York USA

Mark S. Johnson

Alaska Department of Public Health

Juneau, Alaska USA

Mark Klyachko, PhD

Center on Earthquake Engineering

Petropavlovek, Kam, Russia

Radana Konigova, MD

Charles University

Praque, Czech Republic

Per Kulling, MD

Swedish Poison Information Centre

Stockholm, Sweden

John C. Lane, MD

University of Campinas

Medical Center

Sao Paolo, Brazil

Baxter Larmon, PhD, EMT-P

University of CaliforniaLos Angeles Medical Center

Los Angeles, California USA

Todd J. LeDuc

Broward County Commission

Ft. Lauderdale, Florida USA

Prof. Li Zhong-Hao, MD

Beijing Emergency Medical Center

Beijing, China

Susan D. McHenry

National Highway Traffic Safety Administration

Washington, D.C. USA

Sergio Magalini, MD

Unversita Cattolicà del Sacro Cuore

Rome, Italy

Michele Masellis, MD

Osp. Reg. Civico

Palermo, Italy

Eric Noji, MD

World Health Organization

Geneva, Switzerland

Karl-Axel Norberg, MD

Stockholm, Sweden

Judy Olson, BSN, MEd

University of Wisconsin-Madison

Madison, Wisconsin USA

Muneo Ohta, MD

Osaka, Japan
Emergency Care Information Center

Carlsbad, California USA

Paul Paris, MD

Center for Emergency Medicine

University of PittsburghPittsburgh, Pennsylvania USA

Georg Petroianu, MD

University of Heidelberg

Mannheim, Germany

Hugo Prado, MD

Pan-American Health Organization, World Health Organizatior.

RAbdul Radjak, MD

Ministry of Health

Jakarta, Indonesia

Daniel Rodriguez

San Jose, Costa Rica

Lawrence W. Rose, MD

Bethesda, Maryland USA

Leonid B. Roshal, MD

Institute of Pediatrics

Academy of Medical Sciences

Moscow, Russia

Don Schramm

Disaster Management Center

University of Wisconsin

Madison, Wisconsin USA

Knut Ole Sundnes, MD

Baerum Hospital

Oslo, Norway

Frank Thomas, MD

Latter Day Saints Hospital

Salt Lake City, Utah USA

Shao Xiaohong, MD

Peking Union Medical College

Chinese Academy of Medical Science

Beijing, China

Samuel Stratton, MD

Los Angeles County

Los Angeles, California USA

Takashi Ukai, MD

Osaka City General Hospital

Osaka, Japan

Yasuhiro Yamamoto, MD

Department of Emergency and Critical Medicine

Tokyo, Japan

Harsh Wardham, MD

Sundar Lal Jain Hospital

Delhi, India 